\title{
Las nociones de justicia que construyen las niñas en pleno siglo XXI*
}

The notions of justice that girls build in the $2^{\text {lst }}$ century

\author{
Gustavo Adolfo Alarcón Sánchez •• \\ Solman Yamile Díaz Ossa••• \\ Luis Francisco Hernández Méndez••••
}

Fecha de recepción: 12 de septiembre de 2018

Fecha de aprobación: 4 de noviembre de 2018

\section{RESUMEN}

El artículo es producto de la participación en el semillero Justicia y Ciudadanía, que hace parte del proyecto de investigación "Nociones sociales que sobre justicia construyen niños y nińas de colegios oficiales de la localidad de Kennedy en Bogotá D. C.”, financiado por la Fundación Universitaria Los Libertadores. El tema a abordar son las nociones de justicia que

* El presente ensayo es producto del proyecto de investigación: "Nociones sociales que sobre justicia construyen niños y nińas de colegios oficiales de la localidad de Kennedy en Bogotá D. C.”, financiado por la Fundación Universitaria Los Libertadores (Bogotá, Colombia). Citar como Alarcón Sánchez, G., Díaz Ossa, S. y Hernández Méndez, L. (2019). Las nociones de justicia que construyen las niñas en pleno siglo XXI. Via Inveniendi et Iudicandi, 14(1), 67-89. Doi: https://doi.org/10.15332/s1909-0528.2019.0001.03

** Magíster en Educación. Licenciado en Psicología y Pedagogía. Docente de la Facultad de Ciencias Humanas y Sociales de la Fundación Universitaria Los Libertadores (Bogotá, Colombia). gaalarcons@libertadores.edu.co. Orcid: https://orcid.org/0000-0001-7661-5202

*** Magíster en Tecnologías de la Información Aplicadas a la Educación. Licenciada en Educación Especial. Docente de la Facultad de Ciencias Humanas y Sociales de la Fundación Universitaria Los Libertadores (Bogotá, Colombia). sydiazo@libertadores.edu.co. Orcid: https://orcid.org/0000-0001-6442-3625

**** Estudiante de la Licenciatura en Educación Especial de la Fundación Universitaria Los Libertadores (Bogotá, Colombia). Pasante del proyecto de investigación Educación Superior Inclusiva, liderado por la profesora María Angélica Yazzo. Semillerista adscrito al semillero Justicia y Ciudadanía del Programa de Licenciatura en Pedagogía Infantil. lfhernandezm@libertadores.edu.co. Orcid: https://orcid.org/0000-0001-9329-5191 
construyen específicamente las niñas, enfatizado en el conflicto social y personal que ellas enfrentan día a día. El tema se aborda desde la perspectiva de autores como Ralws y Murillo Torrecilla, entre otros, que se ocupan del concepto de justicia y sus características.

El objetivo es visibilizar la justicia entre géneros y motivar la reflexión en el cuerpo docente de preescolar y básica primaria de los colegios en donde se realizó la investigación, para que ejecuten estrategias que permitan a los niños y las niñas comprender la importancia de la mujer en los contextos cercanos en los que viven. Se atiende así a lo estipulado en la Ley 1257 de 2008, "por la cual se dictan normas de sensibilización, prevención y sanción de formas de violencia y discriminación contra las mujeres, se reforman los códigos Penal, de Procedimiento Penal, la Ley 294 de 1996 y se dictan otras disposiciones”.

Palabras clave: justicia, infancia, género.

\section{Abstract}

The article is the product of participation in the research center Justicia y Ciudadanía that is part of the research project "Social notions about justice built by children from official schools of Kennedy's locality in Bogotá DC”. Financed by the Fundación Universitaria Los Libertadores. The topic to approaching is the notions of justice that girls build specifically, emphasized in the social and personal conflict in which they find themselves day by day. The subject is approached from the perspective of authors such as Ralws and Murillo Tordecilla, among others, that deal with the concept of justice and its characteristics.

The article is elaborated in order to make gender justice visible and motivate reflection in preschool and primary school teachers of the schools where the research was conducted to find strategies that allow children to understand the importance of women in the immediate contexts in which they live. It is served well to the stipulated in the Ley 1257 de 2008, "so that standards of awareness are dictated, prevention and sanction of forms of violence and discrimination against women, reform of the criminal codes, of criminal procedure, Ley 294 de 1996 and other dispositions are dictated”.

Keywords: justice, childhood, gender. 


\section{INTRODUCCIÓN}

De conformidad con Sánchez,

La tensión entre neoliberalismo y derechos humanos en América Latina ha generado toda una literatura en la región que ha posibilitado el surgimiento de nuevos marcos analíticos en el derecho como el neoconstitucionalismo, la incorporación en el derecho constitucional de conceptos como el "buen vivir", o el surgimiento de nuevos conceptos como el de Estado constitucional regulador. Este último surge en un contexto de desarrollo económico volcado en la producción de materias primas y la consolidación constitucional de un discurso de los derechos. (2018, p. 96)

Y según Sánchez y Calderón,

Al ser concebidos como universales, los derechos humanos no deberían tener fronteras ni nacionalidad, puesto que están dirigidos a todos los seres humanos. Al ser universales, trascienden las fronteras y al dirigirse hacia la protección de los individuos, se deben dejar a un lado las ciudadanías y/o [sic] nacionalidades. Por lo tanto, son los Estados los que tienen la obligación de garantizar a cualquier individuo su cumplimiento, sin importar si es o no nacional del Estado en que se encuentre. (2017, p. 53)

En concordancia con lo dispuesto en las líneas anteriores,

sin importar dónde nacen, dónde viven, qué raza y sexo tienen, a qué clase social-económica pertenecen, qué ideología política, filosófica o religiosa comparten, si pertenecen a un grupo gitano, indígena o afrocolombiano, todas las personas, por el simple hecho de serlo, son titulares de derechos humanos. En especial forma y calidad, aquellos que les pertenecen a los menores de 18 años de edad (niños, niñas y adolescentes). (Tirado, 2013, p. 148)

La infancia femenina ha sido una población vulnerada, atropellada y violentada desde la antigüedad. Por esta y muchas otras razones este artículo buscará, a través 
de la reflexión, reconocer las barreras y los paradigmas de género que se presentan en la actualidad para pensar una sociedad que acepte la diversidad y la igualdad de derechos de participación y seguridad social.

La literatura sobre infancia ha señalado que solo a partir del siglo XX y gracias a todos los movimientos a su favor y las investigaciones realizadas, se reconoce una nueva categoría: "El niño como sujeto social de derecho" (Jaramillo, 2007, p. 111). A partir de esta concepción se empieza a reconocer que las niñas son un foco de violencia social en estos tiempos. Por ejemplo, en el tema del feminicidio de menores de edad quedan muchos de los casos sin resolución, es decir, sin recibir justicia. Precisamente esa figura hace referencia a la concepción democrática, al principio de eficacia y al de la diferencia en la aplicación de las normas. En este sentido, Rawls afirma:

La concepción democrática mantiene que aun cuando la justicia procesal puede invocarse, al menos hasta cierto grado, la manera en que las interpretaciones previas lo hacen deja todavía un margen excesivo a las contingencias naturales y sociales. Debe notarse, no obstante, que el principio de diferencia es compatible con el de eficiencia, ya que cuando se satisface por completo el primero, es de hecho imposible mejorar a ninguna de las personas representativas sin empeorar a otra, a saber, a la persona representativa de los menos aventajados cuyas expectativas habremos de maximizar. Así, la justicia se define de tal modo que sea congruente con la eficiencia, al menos cuando los dos principios están plenamente satisfechos. Por supuesto que si la estructura básica es injusta, estos principios autorizarán cambios que pudieran reducir las expectativas de algunos de los mejor situados, y por lo tanto, la concepción democrática no será congruente con el principio de eficiencia si este principio es considerado en el sentido de que solo se permiten cambios que mejoren las perspectivas de todos. La justicia tiene primacía frente a la eficiencia y exige algunos cambios que en este sentido no son eficientes. La congruencia se da solo en el sentido de que un esquema perfectamente justo sea también eficiente. (1995, p. 84)

Por otra parte, Murillo y Hernández afirman que 
el anhelo por una mayor justicia social surge, en primer lugar, por la nítida percepción de las múltiples y crecientes injusticias que nos rodean; pero también en la búsqueda de una mejor sociedad. La justicia sobresale, en primer lugar, entre todas las virtudes porque apunta a la rectitud de la voluntad por su propio bien en nuestras interacciones con los demás. (2011, p. 8)

Todas las demás virtudes funcionan ya sea internamente o hacia los demás. La habilidad de intercambiar libre y abiertamente es factor importante en la distribución justa de los bienes de la sociedad. De esta manera, la justicia distributiva es tanto un prerrequisito como un resultado de la justicia conmutativa.

\section{Marco Teórico}

El marco teórico de este artículo se enfoca en dos dimensiones, las instituciones - y su relación con la justicia formal-y las aproximaciones al concepto de infancia, que se abordarán desde diferentes referentes conceptuales y epistemológicos en relación con las nociones de justicia que tienen las niñas y que darán soporte al tema central del artículo. Todo lo anterior bajo el entendido de la necesidad de capacitación de funcionarios y demás operadores para que de esa manera se apropien de los mandatos de la legislación colombiana, los defiendan, los apliquen y los respeten (Bonilla, 2018).

\section{Las instituciones y la justicia formal}

Para comenzar, dentro de la noción de justicia de Rawls existe una relación importante en función de establecer sus principios: "El objeto primario de los principios de justicia social es la estructura básica de la sociedad, la disposición de las instituciones sociales más importantes en un esquema de cooperación” (1995, p. 62).

En ese sentido, Rawls afirma:

Hemos visto que estos principios habrán de gobernar la asignación de derechos y deberes en estas instituciones, y que habrán de determinar la correcta 
distribución de los beneficios y las cargas de la vida social. Los principios de la justicia para las instituciones no deberán confundirse con los principios que se aplican a los individuos y a sus acciones en circunstancias particulares. Estas dos clases de principios se aplican a objetos diferentes que se deben analizar por separado. (1995, p. 62)

De aquí que las políticas públicas se reformulen constantemente de manera abrupta, situación que desfavorece la continuidad de los procesos frente a la aplicación de la justicia de las instituciones garantes y reguladoras de un país. Ahora bien, en algunos casos las instituciones creadas para aplicar las reglas establecidas (como la Procuraduría General de la Nación, la Fiscalía y la Policía Nacional) son la principal fuente de corrupción. Esto es evidencia de los vacíos a nivel de justicia que presenta el marco de la institucionalidad, lo cual incide directamente en la credibilidad y la legitimidad a la hora de aplicar las normas en la sociedad.

En ese orden de ideas, las instituciones tienen un papel fundamental en la sociedad porque toda decisión que tomen repercute directa e indirectamente sobre ellas mismas. Por otro lado, una o varias normas de un acuerdo pueden ser injustas sin que la institución misma lo sea. De igual manera, una institución puede ser injusta, aunque el sistema social en conjunto no lo sea. Rawls afirma:

Podemos, por tanto, distinguir entre una regla única (o grupo de reglas), una institución (o una parte de ella), y la estructura básica del sistema social en conjunto. La razón para hacer esto es que una o varias normas de un acuerdo pueden ser injustas sin que la institución misma lo sea. De igual manera, una institución puede ser injusta aunque el sistema social en conjunto no lo sea. No solo existe la posibilidad de que las normas únicas y las instituciones no tengan en sí mismas bastante importancia, sino que también puede ocurrir que dentro de la estructura de una institución o sistema social, una aparente injusticia se vea compensada por otra. El todo es menos injusto de lo que sería si no contuviese más que una de las partes injustas. Más aún, es concebible que un sistema social pueda ser injusto aun cuando ninguna de sus instituciones sea injusta considerada aisladamente: la injusticia es una consecuencia del modo en que están combinadas dentro de un sistema único. (1995, pp. 64-65) 
Siguiendo a Hart, Ruiz afirma que

las "normas de reconocimiento" de las que habla Bobbio son normas promulgadas, normas válidas del sistema cuya validez se deriva de otras normas (sobre la producción jurídica) conforme a las cuales se han dictado; la regla de reconocimiento de Hart, por el contrario, es una regla última, una regla que no depende de criterios de validez establecidos por ninguna otra regla del sistema, que no es, en consecuencia, jurídicamente válida ni inválida, una regla consuetudinaria que existe solamente como "una práctica compleja, pero normalmente concordante" [...] de identificación del derecho, que opera "como un criterio común y público de decisiones judiciales correctas". (2012, p. 138)

En este orden de ideas, Rawls expone:

El principio de eficiencia puede ser aplicado a la estructura básica mediante referencia a las expectativas de los hombres representativos. Así, podemos decir que un sistema de derechos y deberes en la estructura básica es eficiente si y solo si es imposible cambiar las reglas y redefinir el esquema de derechos y deberes, de modo que se aumenten las expectativas de cualquiera de los hombres representativos (al menos uno) sin que al mismo tiempo se reduzcan las expectativas de algún otro (al menos uno). (1995, p. 76)

Al momento de nacer, el ser humano se convierte en un sujeto de derechos. Eso implica que goza de todas las garantías que tiene cualquier persona. En Colombia, sin embargo, entre los derechos que más se violan están los de las niñas, como se evidencia en el informe anual del Fondo de las Naciones Unidas para la Infancia (Unicef) de 2016 sobre nuestro país en el acápite sobre conflicto armado:

Se evidencia que la edad promedio de reclutamiento de las nińas es 13.4 años de edad y la de los nińos es 14.1 años. La violencia relacionada con el género es difícil de medir y llevar a juicio, especialmente en medio del conflicto armado. Fuentes independientes estiman que entre 2008 y 2012 hubo por lo menos 48914 menores de edad que habían sido víctimas de violencia sexual, 
cuyos presuntos perpetradores eran miembros de grupos armados. Entre estas víctimas, 41313 eran niñas y 7601 niños. (p. 10)

Respecto a ello, la Unicef afirma:

Entre el 2009 y el 2014, 107698 mujeres fueron examinadas por un forense por supuestas agresiones sexuales. Entre ellas, las niñas y adolescentes representaron el grupo más amplio, con el $84 \%$ del número total de casos (94 675). Por lo menos el $26 \%$ de las mujeres y el $15.8 \%$ de los hombres han reportado que golpean a sus hijos como una forma de castigo. El $48.6 \%$ de las mujeres y el $38 \%$ de los hombres han disciplinado a sus hijos con alguna forma de abuso verbal. (2016, p. 11)

Estas cifras componen un panorama apremiante respecto a la necesidad de comprender y operar el marco de la justicia social. Como lo expone Kelsen:

El anhelo por una mayor justicia social surge, en primer lugar, por la nítida percepción de las múltiples y crecientes injusticias que nos rodean; pero también en la búsqueda de una mejor sociedad. El actual movimiento internacional de los Indignados es una muestra de que las tradicionales recetas ya no son suficientes. (Citado en Murillo y Hernández, 2011, p. 8)

Las dificultades que presentan el abordaje y alcance del anhelo de justicia social en casos de violaciones de los derechos de las niñas son evidentes en los procesos judiciales. Si se va a castigar o hacer un proceso justo, hay que tener bases jurídicas fidedignas, que contemplen o apoyen el discurso. Pero en Colombia la persona que vaya a un juzgado o una corte a pelear por una violación de derechos de la mujer requiere más que eso: debe atravesar una maraña de reglas burocráticas que muchas veces juega en contra de las víctimas.

Ahora bien, debemos tener en cuenta que desde la antigüedad a las mujeres y específicamente a las niñas no se las ha visto con igualdad en los ámbitos sociales, políticos o judiciales, entre muchos otros. Esto ha provocado problemas sociales que 
se ven reflejados, por ejemplo, en las instituciones educativas en donde se realizó la investigación. Tomemos en cuenta lo que nos dice Antón Morón:

Que la igualdad y la libertad son aspectos fundamentales; en la vida real o en el contexto real no se da, las feministas han peleado por igualdad de condiciones en todos los aspectos y aunque se ha adelantado [en] muchos [...] aún se sigue viendo la violencia hacia las mujeres y más a las niñas. Particularmente, las mujeres y niñas que no tienen recursos económicos son más vulnerables. (2013, p. 174).

Antón Morón además afirma que existen tres tipos de justicia:

El primer tipo de justicia, la correspondencia de los bienes con la necesidad, se puede contemplar como fundamento de las relaciones familiares o de amistad, del pacto intergeneracional de los adultos respecto de los niños y ancianos, así como de la reciprocidad en las relaciones de pareja; de manera más institucional, es la base normativa de una parte de la acción protectora de los modernos Estados de bienestar. El segundo tipo de justicia está basado en la distribución proporcional al mérito, representa el sistema habitual de remuneración en el empleo: salario igual ante trabajo igual, pero proporcional a la cantidad o calidad — productividad — del trabajo, aspecto central en la remuneración empresarial y en la justificación liberal y marxista. Existe un tercer tipo de justicia, la igualdad distributiva asociada a los derechos humanos: la igualdad de trato, sin discriminación, y la existencia de unos derechos básicos (individuales y colectivos). Ambos aspectos son dependientes de la dignidad del ser humano y como reconocimiento del vínculo social. (2013, p. 181)

Ahora bien, los diversos contextos en los que está involucrado el sujeto y las dinámicas que se generan en estos determinan las características para la construcción de su identidad, sin dejar de lado que las interacciones sociales posibilitan el reconocimiento de su individualidad. Si nos trasladamos a la vida escolar, los principios de la justicia se han mantenido estáticos y poco usados por la comunidad educativa: el concepto de justicia se ha leído, pero no se ha aplicado. Así se genera en vez de igualdad, 
desigualdad; en vez de equidad, inequidad; en vez de justicia, injusticia, y así sucesivamente. Como lo plantea Bolívar:

Justicia, igualdad y equidad, a menudo empleados indistintamente, conceptualmente se enmarcan en la actualidad de paradigmas diferenciados. Si desde el paradigma de la igualdad todos los individuos deben siempre recibir el mismo tratamiento; desde el marco de la equidad los individuos son diferentes entre sí y merecen, por lo tanto, un tratamiento diferenciado que elimine o reduzca la desigualdad de partida. La contraposición es manifiesta cuando, desde el marco de la equidad, el tratamiento desigual es justo siempre que pueda beneficiar a los individuos más desfavorecidos. La universalización está vinculada al paradigma de la igualdad: todas las personas son iguales ante la ley, mereciendo los mismos derechos y recursos. (2012, p. 12)

Bajo estas perspectivas vale la pena atender al principio de la diferencia (una forma de principio de equidad) que expone Rawls como lo cita Bolívar:

Se basa en la idea de que individuos desiguales deben ser tratados de modo desigual para que dicha desigualdad sea reducida. En el ámbito educativo, como se comentará, la igualdad de oportunidades es expresión del primero [el principio de igualdad], determinadas propuestas compensatorias lo son de la equidad. Sin embargo, en la práctica y desde las teorías de la justicia, como se verá, el asunto no es simple, como si se pudiera contraponer de modo general uno a otro. Se puede defender, como hace Rawls, una igualdad en la distribución de algunos bienes primarios y equidad en la distribución de otros, aplicándose uno y otro a esferas o conjuntos de bienes distintos. (2012, p. 12)

En esa línea tiene un sentido importante la justicia social como referente a tener presente en el abordaje de la población infantil femenina:

Viene dada por prácticas y condiciones sociales que posibilitan el reconocimiento mutuo con atención afectiva, igualdad jurídica y estima social. Hay formas de trato socialmente injustas en las que lo que está en juego no es distribución de 
bienes o derechos, sino ausencia de afectos y cuidado o de estima social, que hurtan la dignidad o el honor. (Bolívar, 2012, p. 27)

\section{Infancia}

Como otro elemento de la reflexión teórica, hablar de infancia implica reconocer una gama de complejidades. Pavez remite a un concepto enunciado por la Real Academia Española (RAE):

Los conceptos de infancia y niñez presentan una gran complejidad no solo a nivel social, sino desde la propia etimología de estas palabras. Según la Real Academia de la Lengua Espańola (RAE), "Infancia" proviene del latín infantia, cuyo significado primario alude a la incapacidad de hablar y define a los înfâns o înfantis como aquellos que no tienen voz. Para la RAE actualmente la infancia es delimitada como: i) el periodo de la vida humana desde que se nace hasta la pubertad; ii) el conjunto de los niños de tal edad; y iii) el primer estado de una cosa después de su nacimiento o fundación. Por otro lado, la RAE define al infante como: i) el niño que aún no ha llegado a la edad de siete años; ii) el pariente del rey que por gracia real obtiene el título de infante o infanta; y iii) cada uno de los hijos varones y legítimos del rey, nacidos después del príncipe o de la princesa. (2012, p. 82)

Pavez resalta que el concepto de infancia se asume como unidad de análisis que requiere profundizar en aproximaciones investigativas que tengan presente la familia como escenario fundamental en su construcción. En esta línea de argumentos se puede ubicar a Émile Durkheim, quien, interesado en la sociología de la educación, desarrolla un pensamiento sobre la infancia como un fenómeno presocial: "Un terreno casi virgen donde se debe construir partiendo de la nada y poner en ese lugar una vida moral y social" (citado en Pavez, 2012, p. 83).

El ser humano en su estado de infante tiene conocimientos de comunicación: el llanto, por ejemplo, es una manera en la que se comunica con su madre y establece 
una relación con su entorno. Ese conocimiento va madurando durante los años y lo lleva a un estado presocial que utiliza para construir sus criterios sociales y morales.

\section{Para DeMause,}

a primera vista esta falta de interés por la vida de los niños resulta extraña. Los historiadores se han dedicado tradicionalmente a explicar la continuidad y el cambio en el transcurso del tiempo, [...] y desde Platón se ha sabido que la infancia es una de las claves para ello. No se puede decir que fuese Freud quien descubrió la importancia de las relaciones padre-hijo para el cambio social. (1974, p. 3)

En esa perspectiva, como lo expone en primer lugar Pavez, se presenta frente a las niñas una construcción de estereotipos:

El hecho de asociar la infancia a un estado más cercano a la naturaleza que a la cultura -al igual que ocurre con otras minorías políticas, como las mujeres o los grupos indígenas- construye un estereotipo generacional sobre las niñas y los niños como seres inferiores, que necesariamente deben estar situados bajo el poder y la autoridad de una persona adulta, la cual se considera racional y civilizada. (Pavez, 2012, p. 84)

Y en segundo lugar, como lo delimita Alzate, frente a la infancia hay una construcción conceptual distanciada de una infancia viva y real:

Si bien la sociología ha estudiado al nińo como elemento social, la pedagogía como sujeto de educación y escolarización; y la psicología como sujeto de desarrollo fisiológico y psicológico, la historia lo ha hecho de una manera incidental; la prueba está en que el mismo "concepto de infancia" podría ser una expresión que explica el distanciamiento de la infancia viva y real. (Alzate, 2003, p. 22)

Bajo esta perspectiva, se infiere que las representaciones de la infancia, independientemente de que se trate de niños o niñas, dan cuenta de sujetos 
dependientes que obedecen a un poder impuesto por un adulto con más experiencia, que orienta de forma positiva o negativa. Otra perspectiva que permite hacer una lectura de la infancia se ubica en la teoría funcionalista de Parsons, que afirma:

Las niñas y los niños son vistos como receptores pasivos de los contenidos que la sociedad considera necesarios para que una persona se convierta en adulta. Se trata de los mecanismos que reproducen el orden social en el seno de una sociedad funcionalista. Desde el momento del nacimiento - e incluso antes del nacimiento, en términos de género- se depositan demandas y exigencias a través de la asignación de un determinado rol a las nińas y los niños. (Citado en Pavez, 2012, p. 85)

Teniendo en cuenta lo anterior, se deduce que la infancia no tiene voz en las decisiones que se toman en sus sistemas micro (casa) y meso (contexto cercano), ya que la cultura educa a niños y niñas en la obediencia y el acatamiento de órdenes, sin presentarse alternativas de participación. En este orden de ideas, el deber ser no solamente es una cuestión generacional, la obediencia que deben mostrar las niñas y los nińos hacia las normas que les enseñan las personas adultas, sino que "se trata de normas establecidas en términos de género, ya que justamente es durante la infancia cuando se acelera e intensifica el proceso de aprendizaje de los roles de género y las relaciones de poder entre los géneros" (Pavez, 2012, p. 86).

Desde la antigüedad se ha visto al niño o la niña como un sujeto que no puede ser plenamente consciente de sus acciones. En efecto, la niñez es un periodo con pocos conocimientos en cuanto a reglas sociales contextuales, salvo los predispuestos desde el vientre de la madre. Sin embargo, se escucha la expresión popular "Los niños de ahora vienen con el chip encendido", que puede entenderse como que es inevitable la libre expresión en la niñez (independientemente cual sea el código de comunicación).

Por su parte, el enfoque constructivista afirma que

el ser humano es un producto social y todo su desarrollo está socialmente construido e interferido, incluso la infancia. Para estos autores, en la construcción social de la realidad se registran los momentos de externalización, 
objetivación e internalización a través del proceso de socialización primaria y secundaria donde se internaliza la realidad objetiva. (Berger y Luckmann, 1968. p. 39)

La cultura capitalista en la actualidad también ha tenido un valor importante en la concepción de infancia: como el niño o la niña no tiene una entrada monetaria, no tiene derecho sobre el adulto; como el adulto cubre económicamente las obligaciones en la casa, es el que tiene el mandato y toma las decisiones:

Por otro lado, la dependencia económica que afecta a la niñez moderna agudiza su condición de minoría respecto al poder adulto, puesto que en el sistema capitalista el hecho de obtener y disponer de dinero significa un mecanismo —aunque no el único — de empoderamiento y capacidad de tomar decisiones. Esta situación de desventaja económica lleva a Oldman [...] a sugerir que las relaciones generacionales entre el colectivo infantil y el adulto puedan leerse como relaciones de clase social, cuyo estatus económico favorable lo tienen las personas adultas. (Pavez, 2012, p. 92)

Por su parte, Alzate plantea que

las nuevas formas de distribución del poder social exigirán modos específicos de educación de los niños quienes dejarán, y esto es válido solo para los hijos de los grupos con recursos, de ser socializados directamente por la comunidad, de aprender el oficio de sus mayores... (2003, p. 47)

Como se podrá apreciar en el apartado "Resultados", las niñas se asumen como las sucesoras de sus madres, encargadas de los oficios de la casa. Como lo afirma Palacio:

Las madres por su parte enseñaban a sus hijas desde muy temprana edad a realizar las actividades femeninas, tales como la hilandería, la cocina, entre otros. Por ejemplo, una niña de 5 años de edad debía aprender a manejar el huso. $(2004$, p. 4) 
Por ello, toma sentido la perspectiva expuesta por la sociología de la infancia, que muestra el paradigma social asociado a esta población: las niñas y los niños se asumen como agentes pasivos de una sociedad adulta que los agrede por muchos factores. Entre estos tenemos el capitalismo, que limita al niño o niña porque el adulto tiene dinero y como actualmente el dinero es sinónimo de poder, la niñez tiene desventajas.

Es importante reconocer que hay diferentes tipos de infancias. No es lo mismo la niñez en la zona rural que la niñez en la zona urbana. De hecho, dentro de tales grupos también hay diferencias dependiendo del contexto. Hay muchos factores que influyen en la construcción de las infancias.

\section{CONCEPCIÓN DE NIÑA}

Las niñas desde su nacimiento son sujetos de derechos y, en cuanto tales, seres sociales únicos, irreductibles, activos y con subjetividad propia. Esta concepción implica que se consideran seres humanos dignos, amparados por los derechos propios a su naturaleza y particular momento del ciclo vital. (Comisión Intersectorial para la Atención Integral de Primera Infancia, 2013, p. 15)

En ese orden de ideas, las niñas tienen una noción de justicia, pero es un poco débil, basada en la propia experiencia de sus sistemas micro y meso.

Para los especialistas, la educación no es cosa de laboratorio, de instituto, de experimento científico, sino una cosa de amor que necesita ante todo el calor que únicamente nace en el seno de la familia, la cual es para la niña un anticipo de lo que será el mundo y la sociedad para la mujer. Dentro de la familia la nińa debe aprender esta lucha constante consigo misma y con las circunstancias que ha de conducirle con alegría y con inteligencia a cumplir con sus objetivos. (Jiménez, 2008, p. 26) 
Sin embargo, como lo exponen Atehortúa y Rojas respecto al caso de Carmen Rodríguez,

lastimosamente, casi nadie en Colombia sabe del papel definitivo que las mujeres jugaron el día en que empezó a nacer nuestra nación. Una mujer de la cual poco se conoce, Carmen Rodríguez, fue acusada como abanderada del movimiento y desterrada por esa causa en 1816. Sin embargo, no hay en la Plaza de Bolívar o en la Casa Museo del 20 de Julio un monumento que la recuerde. La placa conmemorativa solo menciona a un "excelso grupo de patriotas"...todos ellos hombres. (2005, p. 273)

Lo anterior evidencia una relación dinámica entre los conceptos de justicia e infancia, tal que la justicia se muestra como un conjunto de normas sociales que regulan la interacción entre seres humanos y permite así comprender lo justo e injusto del contexto que rodea a la niñez.

\section{MÉTOdoS Y MATERIALES}

Se utilizó el enfoque cualitativo a partir del método interpretativo, lo que permitió objetivar las subjetividades, enunciaciones y representaciones elaboradas por las niñas respecto a la justicia y los conflictos presentes en su contexto. La temática abordada fue la de las nociones de justicia que construyen las niñas, enfatizado en los conflictos sociales y personales en los que se ven inmersas día a día. Esas nociones se contrastaron con las de los autores de referencia.

La muestra que se escogió estuvo conformada por veinticuatro nińas del Colegio Distrital la Amistad, de los cursos 201 y 202, que habitan en la Localidad de Kennedy, en barrios como Timiza, Carvajal, Las Américas, Bavaria, Castilla, Tíntal Norte, Calandaima, Corabastos, Patio Bonito, Las Margaritas, entre otros.

Se organizó con esta población una serie de talleres en el marco del proyecto macro, orientados por los siguientes abordajes: conocer las normas institucionales de convivencia y las normas de la familia, reconocer desde la cartografía social 
las problemáticas vividas en sus contextos inmediatos, participar en un taller notiescolar para identificar lo justo e injusto y elaborar diarios de campo sobre todo ese trabajo. A partir de estos últimos se desarrolló una lectura de los registros resultantes de los talleres que permitieron identificar las representaciones de justicia de las niñas.

\section{Resultados}

Dentro de las nociones a destacar se presenta una idea de justicia respecto al género que denota la vulnerabilidad de la condición de mujer, representada en situaciones explicitas de violencia de género. Un ejemplo de ello se evidenció en los dibujos elaborados en el taller de cartografía social (figuras 1 y 2).

Figura 1. Taller de cartografía social

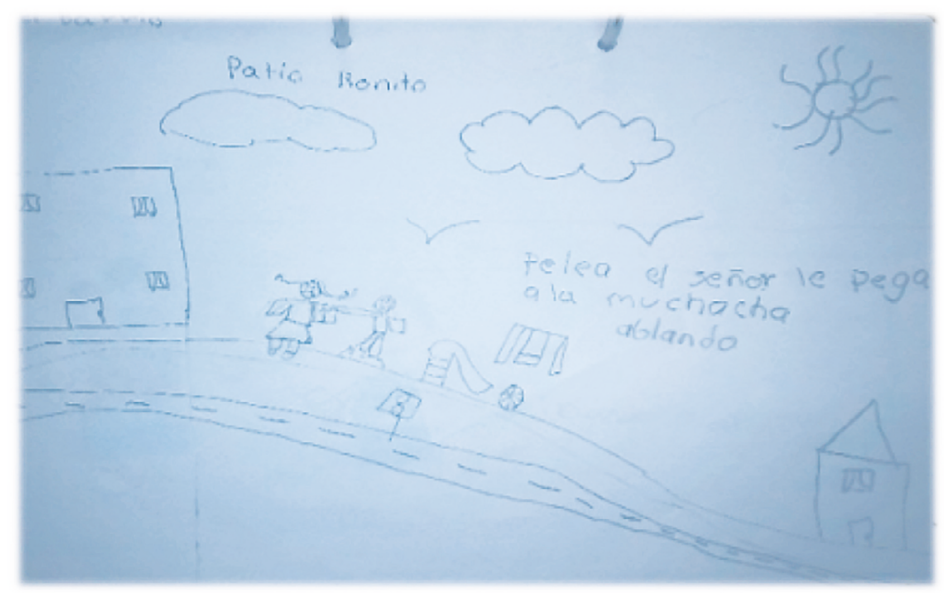

Fuente: archivos fotográficos proyecto macro "Nociones sociales que sobre justicia construyen niños y niñas de colegios oficiales de la localidad de Kennedy en Bogotá D. C.”.

En los dibujos de las niñas se evidencia conocimiento del maltrato que sufre la mujer en su contexto. Además, una nińa exponía en las reflexiones del taller de normas de 
familia: "Mi papá le ha pegado a mi mamá", lo que muestra el panorama de violencia en sus entornos familiares. Otro referente interesante mostró que en ese mismo entorno se asigna a las nińas un rol y una responsabilidad adquirida en labores del hogar que denotan una diferenciación frente a los niños.

Respecto al taller de normas institucionales de convivencia, se evidencióla participación de la mujer en el colegio advirtiendo que se da más vocería y participación a los niños que a las niñas.

Figura 2. Taller de cartografía social

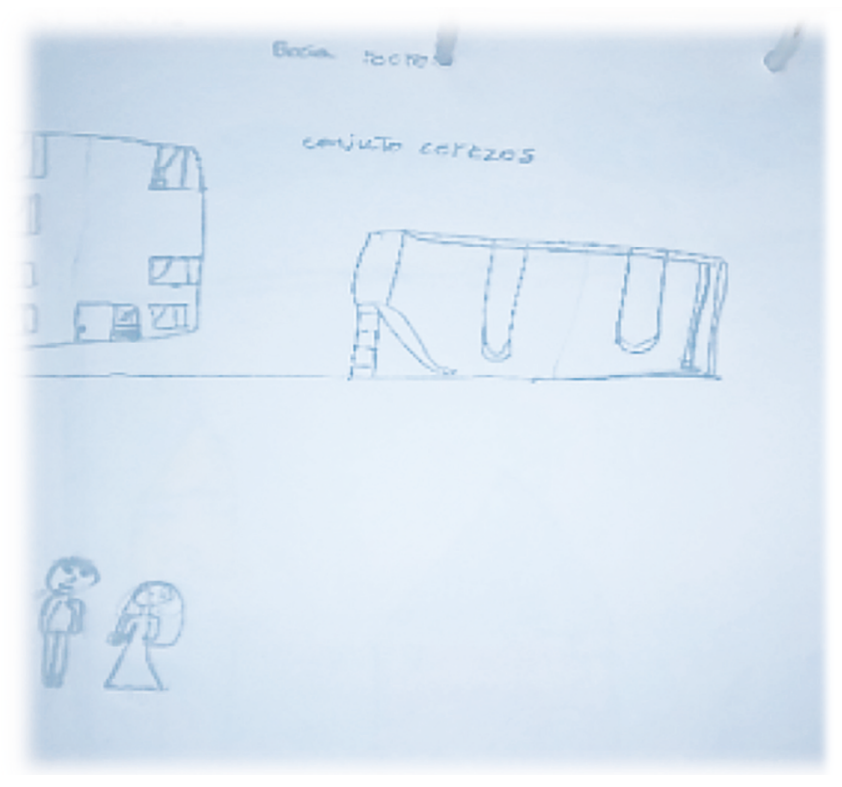

Fuente: archivos fotográficos proyecto macro "Nociones sociales que sobre justicia construyen niños y niñas de colegios oficiales de la localidad de Kennedy en Bogotá D. C.”.

Respecto a los registros realizados por medio del diario de campo, se observó que constantemente en el grupo se exponen situaciones de violencia. Un ejemplo de ello se dio en el taller de notiescolar: 


\begin{abstract}
Algunos leyeron los registros en los que se hizo referencia a los últimos acontecimientos de las injusticias que están viviendo los venezolanos a causa de la dictadura: "Es cruel que maten a los hombres", "Las calles están con candela", "Les pegan”. Además, hubo registros como el siguiente: “A una mujer le robaron la bicicleta y nadie hizo nada. (Diario de campo, 2017)
\end{abstract}

Se relacionaron algunos ejemplos de conflicto en los que las niñas se presentaban como protagonistas de vulneración: "Un día una niña peleó con una niña” (como solución propusieron que se pidieran disculpas) y "Un niño estaba obligando a una niña a que le diera un beso y ella no quería” (Diario de campo, 2017).

Así pues, las nociones que se exponen a nivel de justicia de género visibilizan la vulneración puesto que se lee a la infancia como receptora pasiva de las consecuencias de la acción de la sociedad, que solo asume el papel de escucha y obediencia, sin permitírsele un conocimiento crítico reflexivo de su entorno familiar, escolar y social.

Desde esta perspectiva la justicia muy poco aplica a las nińas, y si aplica no les ofrece suficientes garantías para romper los prejuicios sociales aún vigentes. Las niñas en la actualidad tienen una noción de justicia un poco débil, basada en su experiencia de los niveles micro y meso del sistema. De hecho, a nivel familiar la niña es la sucesora de la madre y es quien se encarga de ser buena esposa y madre. $\mathrm{Al}$ respecto es importante no olvidar que al constituirse Colombia como Estado social de derecho su objetivo es la protección del más débil jurídicamente hablando (Manzo, 2018).

\title{
CONClusiones
}

De acuerdo con los resultados obtenidos durante el proceso de la investigación, a continuación se presenta la discusión teórica con relación a las categorías encontradas (género y vulnerabilidad femenina y violencia contra la mujer especificamente en la infancia). 
Tal como lo plantea la Comisión Intersectorial (2013), la primera infancia tiene derechos cuyo cumplimiento debe ser verificado. Los adultos debemos ser veedores de ellos y construir redes que afiancen las características sociales de estos sujetos. Por esta razón resulta imperativo comprender la infancia y nuestra responsabilidad en cuanto a su desarrollo, amparar los derechos inherentes a su naturaleza y comprender las particularidades de esa etapa vital.

Asimismo, las decisiones que tomen los adultos en cuanto a políticas públicas para las nińas deben incorporar un reconocimiento a su dignidad. En esto radica abordar apropiadamente las relaciones y las decisiones que contribuyen, de forma directa o indirecta, a asegurar las condiciones deseables para su crecimiento y desarrollo. Por lo anterior es imperativo reconocer las formas de vulneración de los derechos de la infancia femenina.

Unicef, por ejemplo, afirma que "la violencia basada en género, especialmente la violencia sexual contra las niñas, constituye una amenaza crítica, a pesar de que la incidencia está enormemente subrreportada" (2016, p. 10). Y aunque esta violencia sexual, sicológica y física contra la mujer específicamente en la infancia ha sido expuesta a la luz pública en todos los medios de comunicación que actualmente utilizamos, no parece haberse alcanzado una sensibilización suficiente para disminuirla. Vale la pena no olvidar las cifras expuestas en el informe de la Unicef de las mujeres que fueron examinadas por un forense por supuestas agresiones sexuales. Es importante tener en cuenta estas cifras, ya que muestran las amenazas a las que se ven expuestas las nińas y dimensionan las realidades en las que lamentablemente son protagonistas. Por ello, es importante recalcar la comprensión que se debe tener respecto a la violencia contra la mujer:

Por violencia contra la mujer se entiende cualquier acción u omisión, que le cause muerte, daño o sufrimiento físico, sexual, psicológico, económico o patrimonial por su condición de mujer, así como las amenazas de tales actos, la coacción o la privación arbitraria de la libertad, bien sea que se presente en el ámbito público o en el privado. (República de Colombia, Ley 1257 de 2008, p. 1) 
Finalmente, identificar los conceptos de justicia y de infancia permite comprender las realidades a las que se enfrentan las niñas en pleno siglo XXI. En consecuencia, es imperativo que la Educación y la Pedagogía aporten reflexiones y acciones que visibilicen tales realidades para que la violencia disminuya y en el aula estudiantil florezcan relaciones asertivas entre géneros.

Por consiguiente, la escuela, como escenario de socialización y entorno de justicia, tiene la responsabilidad de asumir un papel protagónico en el fortalecimiento de los derechos de las niñas, puesto que en ella se encuentran la mayor parte de su tiempo. Es imperativo el reconocimiento de la diversidad cultural y de género que cohabita en las instituciones educativas, a través del fortalecimiento de prácticas pedagógicas vinculantes que reconozcan la diferencia. En otras palabras, debe comprenderse que educar y proporcionar equidad de género puede minimizar la vulneración de los derechos infantiles especialmente en las niñas.

\section{REFERENCIAS}

Alzate, M. (2003). La infancia: concepciones y perspectivas. En La infancia: concepciones y perspectivas. S. L.: S. E.

Antón Morón, A. (2013). Igualdad y libertad: fundamentos de la justicia social. Revista Internacional de Educación para la Justicia Social, 2(1), 173-195.

Atehortúa, A. y Rojas, D. (2005). Mujer e historia. Revista Cientifica, 7, 269-293. Recuperado de https://goo.gl/REX5Q6

Berger, P. L. y Luckmann, T. (1968). La construccion social de la realidad. Buenos Aires: Amorrortu.

Bolívar, A. (2012). Justicia social y equidad escolar. Una revisión actual. Revista Internacional de Educación para la Justicia Social, 1(1), 9-45. 
Bonilla, H. (2018). Sugerencias para la primera reforma al Código General del Proceso (Ley 1564 de 2012). Via Inveniendi et Iudicandi, 13(1), 217-236. Doi: http://dx.doi. org/10.15332/s1909-0528.2018.0001.08

Comisión Intersectorial para la Atención Integral de Primera Infancia (2013). Fundamentos técnicos de la Estrategia de Atención Integral a la Primera Infancia. Recuperado de https:// goo.gl/jGQGyi

DeMause, L. (1974). La evolución de la infancia. En La evolución de la infancia. S. E.: S. L. Recuperado de https://goo.gl/LdBdLo

Jaramillo, L. (30 de noviembre de 2007). Concepción de infancia. Recuperado de https:// www.redalyc.org/html/853/85300809/

Jiménez, A. (27 de junio de 2008). Historia de la infancia en Colombia: crianza, juego y socialización, 1968-1984. Anuario Colombiano de Historia Social y de la Cultura, 35, 155-188. Recuperado de https://goo.gl/RXyh1v

Manzo, G. A. (2018). Análisis comparado de una sentencia relativa al Estado social de derecho del Tribunal Supremo de Justicia venezolano (TSJ) y la Corte Constitucional Colombiana (CCC). Verba Iuris, 13(40), 175-187.

Murillo Torrecilla, J. y Hernández Castilla, R. (2011). Hacia un concepto de justicia social. Revista Iberoamericana sobre Calidad, Eficacia y Cambio en Educacion, 9(4), 7-23.

Palacio, C. (octubre de 2004). El descubrimiento de América, pasado y porvenir de la infancia de este continente. Recuperado de https://goo.gl/9X6YjG

Pavez, I. (2012). Sociología de la infancia: las niñas y los niños como actores sociales. Revista de Sociología, 27, 81-102.

Rawls, J. (1995). Teoria de la justicia. México: FCE. Recuperado de https://etikhe.files.wordpress.com/2013/08/john_rawls_-_teoria_de_la_justicia.pdf 
República de Colombia, Congreso de la República, Ley 1257 de 2008. Diario Oficial, 47193 (4 de diciembre).

Ruiz, J. (2012). Bobbio y los conceptos de norma jurídicamente última. Sequência (Florianópolis), 64. Recuperado de https://goo.gl/KTmVfJ

Sánchez, R. (2018). La regulación de participación. El caso de la consulta previa en Colombia, Perú y Chile. Via Inveniendi et Iudicandi, 14(1), 95-118. Doi: http://dx.doi. org/10.15332/s1909-0528.2018.0001.03

Sánchez, C. y Calderón, D. (2017). Geopolítica de los derechos humanos en las fronteras terrestres de Colombia en el primer periodo presidencial de Juan Manuel Santos. Via Inveniendi et Iudicandi, 12(2), 53-72. Doi: http://dx.doi.org/10.15332/ s1909-0528.2017.0002.02

Tirado, M. (2013). Niños, niñas y adolescentes en el conflicto armado en Colombia. Verba Iuris, 29, 147-159.

Unicef Colombia (2016). Informe anual 2016. Recuperado de https://goo.gl/b48Um4 